\title{
UM MODELO PARA ANALISAR O PROBLEMA DE FILAS EM CAIXAS DE SUPERMERCADOS: UM ESTUDO DE CASO
}

\author{
Reinaldo Morabito \\ Flavio C. R. de Lima \\ Departamento de Engenharia de Produção \\ Universidade Federal de São Carlos \\ 13565-905 São Carlos, SP \\ (morabito@power.ufrscar.br, \\ flavio.lima@hotmail.com)
}

\begin{abstract}
Resumo
Neste estudo aplicamos teoria de filas para analisar o problema de congestão em caixas de supermercados. Estamos particularmente interessados em modelar o tempo médio de espera em fila, aqui entendido como uma componente importante do nível de serviço ao consumidor, em função da capacidade do sistema (i.e., o número de caixas num dado período de tempo). Três modelos são explorados: (i) representar o sistema por meio de um simples modelo $\mathrm{M} / \mathrm{M} / m$ de fila única, onde $m$ é o número de caixas, (ii) representar o sistema por meio de $m$ modelos $\mathrm{M} / \mathrm{M} / 1$ paralelos e independentes, e (iii) representar o sistema por meio de um modelo Markoviano mais geral, onde os dois modelos anteriores podem ser vistos como casos particulares. Para avaliar o desempenho destes modelos, realizamos um estudo de caso num dos supermercados da rede Jaú-Serve, localizado no interior de São Paulo. Os resultados mostraram que o terceiro modelo pode resultar numa boa aproximação para o problema, ao contrário dos dois primeiros.
\end{abstract}

Palavras chave: teoria de filas, supermercados, modelo Markoviano.

\begin{abstract}
In this study we applied queueing theory to analyze the problem of congestion in supermarket checkouts. We are particularly concerned with modeling the mean user waiting time in the queue, viewed as an important component of the user service, as a function of the capacity of the system (i.e., the number of cashiers in a given time period). Three models are explored: (i) to represent the system by means of a simple $\mathrm{M} / \mathrm{M} / m$ queueing model, where $m$ is the number of cashiers, (ii) to represent the system by means of $m$ parallel and independent $\mathrm{M} / \mathrm{M} / 1$ queueing models, and (iii) to represent the system by means of a more generic Markovian model, where the former models can be viewed as particular cases. In order to evaluate the performance of the models, we developed a case study in one of the supermarkets of JaúServe chain located in São Paulo State. The results showed that the third model can yield a good approximation for the problem, different from the first two ones.
\end{abstract}

Keywords: queueing theory, supermarkets, Markovian model. 


\section{Introdução}

No Brasil, o setor de supermercados vem crescendo significativamente nos últimos anos, mas ainda tem a característica de ser um setor regional e fragmentado. Os varejistas de comida in natura que operam no sistema self-service detêm aproximadamente $84 \%$ do total de vendas do setor, o que é uma alta porcentagem para a América Latina (Andersen, 1997). De acordo com a Associação Brasileira de Supermercados (ABRAS), este percentual corresponde a cerca de US\$44 bilhões, ou 6,6\% do PIB brasileiro (Superhiper, 1998). As duas maiores companhias do setor detêm $18 \%$ do mercado, enquanto que as vinte maiores detêm $58 \%$. Para efeito de comparação, na Alemanha, por exemplo, as cinco maiores redes de supermercados já respondem por $70 \%$ do mercado. Pode-se observar uma tendência a lojas grandes fazendo uso de tecnologias avançadas, menor número de empregados, e maior número de caixas de atendimento.

Para aumentar o volume de vendas, gerentes de supermercados têm se preocupado em melhorar o nível de serviço ao consumidor. De acordo com uma pesquisa realizada em 1996 na grande São Paulo (OMC/EAN, 1996), o consumidor em geral é fiel ao supermercado que compra, pois, segundo os entrevistados, o costume leva ao conhecimento do espaço físico, da distribuição dos produtos, e dos horários menos congestionados do supermercado. Os clientes de supermercados mais automatizados valorizam, como primeira opção, o atendimento rápido e funcionários competentes e educados. Por outro lado, os clientes de supermercados menos automatizados valorizam mais o preço e a proximidade do supermercado. Em ambos os casos, preços e filas exagerados são apontados como os fatores mais negativos.

Ainda segundo esta pesquisa, é interessante notar que os consumidores correlacionam o tempo total gasto no supermercado com a falta de organização geral do supermercado. Os consumidores consideram que economizam tempo com uma boa disposição de produtos nas gôndolas, presença de preços nos produtos e atendimentos mais automatizados nos caixas, mas apontam como o maior problema a ser atacado o tempo de espera nas filas dos caixas. Desta maneira, uma preocupação dos gerentes de supermercados é como reduzir o tamanho das filas nos caixas. Ao aumentar o número de caixas em operação, o gerente estará reduzindo o tempo médio de espera em fila, por outro lado, também estará aumentando os custos operacionais do sistema. Surge então um interessante tradeoff a ser analisado entre o nível de serviço a ser oferecido ao cliente e a escolha de capacidade do sistema de atendimento.

No presente trabalho estamos interessados em modelar o tempo médio de espera em função da capacidade do sistema, por meio de teoria de filas. Três modelos são explorados: (i) representar o sistema por meio de um simples modelo $\mathrm{M} / \mathrm{M} / m$ de fila única, onde $m$ é o número de caixas em operação, (ii) representar o sistema por meio de $m$ modelos $\mathrm{M} / \mathrm{M} / 1$ paralelos $\mathrm{e}$ independentes, e (iii) representar o sistema por meio de um modelo Markoviano mais geral, onde os dois modelos anteriores podem ser vistos como casos particulares. Para avaliar o desempenho destes modelos, realizamos um estudo de caso num dos supermercados da rede Jaú-Serve, que atua no interior de São Paulo. Os resultados apresentados na seção 5 mostram que o terceiro modelo, apesar de demandar um esforço computacional maior do que os anteriores, é bem mais apropriado para descrever o sistema.

\section{Definição do problema}

Poucos trabalhos foram encontrados na literatura analisando o problema de congestão em caixas de supermercados. Umesh et al (1989) estudaram a sensibilidade dos consumidores com relação ao tempo de espera num supermercado. Um consumidor que ficar insatisfeito com o 
tempo de espera, embora possa não desistir de efetuar a compra, pode não voltar mais ao supermercado. O problema abordado foi o de maximizar a margem de contribuição, definida como o ganho $R(L)$ menos o custo $V(L)$, onde $L$ é a taxa de demanda do consumidor em função do tempo médio de espera. O procedimento foi aplicado para determinar o número de caixas numa filial de uma rede de supermercados na Nova Inglaterra, EUA. Ao aumentar o número de caixas de 6 para 10 (nos horários de pico), o tempo médio de espera foi reduzido de 34 minutos para menos de 15 minutos. Conforme os autores, o volume de vendas cresceu $19 \%$, e as reclamações dos clientes diminuíram em cerca de $40 \%$. Os ganhos reais cresceram 17\%, justificando os custos adicionais do aumento do número de caixas.

Outro estudo interessante sobre congestão em caixas de supermercados foi encontrado em Dijk (1997). O artigo analisou a aplicação de uma alocação flexível da capacidade em um supermercado na Holanda, para reduzir as flutuações no atendimento da demanda e balancear os workloads dos caixas. O número de consumidores neste supermercado passou a ser eletronicamente controlado ao longo do tempo e, em função deste número, caixas iam sendo abertos ou fechados conforme os resultados de um modelo baseado em teoria de filas. $\mathrm{O}$ supermercado adotou uma estratégia de dar descontos especiais aos consumidores que não encontrassem caixas com menos de três consumidores em fila. Conforme Dijk (1997), esta estratégia resultou num custo adicional de $2 \%$ do valor das vendas, enquanto que as vendas cresceram $20 \%$. Uma pesquisa de opinião junto aos consumidores indicou que este aumento das vendas deveu-se mais à redução dos tempos de espera dos consumidores, do que à possibilidade deles obterem descontos especiais.

No presente trabalho, admitimos que não sabemos como a demanda de consumidores do supermercado varia em função do tempo médio de espera em fila, diferente do estudo em Umesh et al (1989). Também supomos que não dispomos de informações on-line sobre o número de consumidores no supermercado ao longo do tempo, diferente do estudo em Dijk (1997). Os caixas são separados em caixas normais e caixas rápidos, estes últimos atendem exclusivamente consumidores com até 10 itens. Os caixas normais atendem em geral consumidores com mais de 10 itens, embora também possam atender consumidores com menos itens. Para cada tipo de caixa, admitimos que os consumidores chegam durante um dado período de tempo conforme um processo de Poisson. Os tempos de serviço, tanto dos caixas normais quanto dos rápidos, são supostos exponencialmente distribuídos (estas hipóteses são analisadas na seção 4).

Consideramos que os consumidores, ao chegarem no sistema, podem desistir de comprar e sair do supermercado, se as filas dos caixas estiverem muito grandes ou demoradas. Devido a limitações de espaço na frente dos caixas e para reduzir as dificuldades para movimentar carrinhos carregados de compras, os consumidores aguardam atendimento na fila de um dos caixas, ao invés de em uma fila única, como acontece em outros sistemas como bancos, correios, etc. Também consideramos que os consumidores, ao chegarem, escolhem sempre o caixa com menor fila, e que mudam de fila durante o tempo de espera, dependendo do tamanho da fila dos outros caixas.

\section{Modelagem por meio de teoria de filas}

Admitindo-se que os intervalos de tempo entre chegadas de consumidores e os tempos de serviço dos caixas sejam exponencialmente distribuídos, os dois candidatos mais simples da teoria de filas para modelar o sistema de atendimento dos caixas de supermercados são:

(i) um modelo $M / M / m$ com fila única e disciplina de atendimento FCFS (First Come, First Served), onde $m$ é o número de caixas (normais ou rápidos). Consideramos que $\lambda$ 
consumidores por unidade de tempo chegam em média no sistema, e que os $m$ caixas em paralelo são iguais, cada um com capacidade média de $\mu$ consumidores por unidade de tempo (figura 1a).

(ii) $\quad m$ modelos $M / M / 1$ paralelos e independentes, cada um com disciplina de atendimento FCFS. Consideramos que $\lambda / m$ consumidores por unidade de tempo chegam em média em cada caixa, e que cada um dos $m$ caixas tenha capacidade $\mu$ (figura 1b).

Sistema de Fila Única

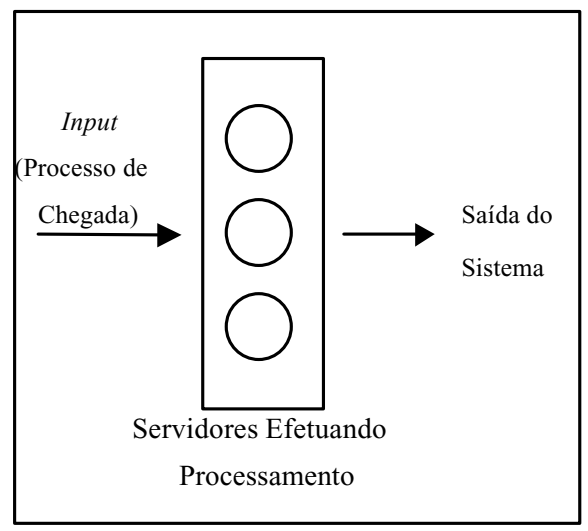

(a)
Sistema de Filas em Paralelo

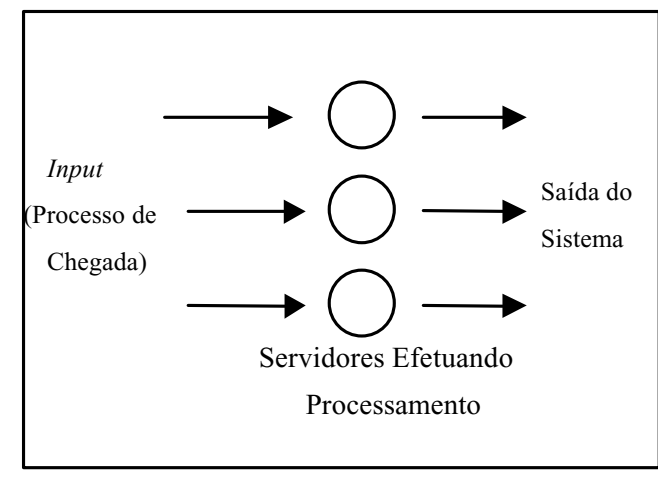

(b)

Figura 1 - (a) Sistema de fila única (modelo $M / M / m$ ) e (b) sistema de filas paralelas e independentes $(m$ modelos $M / M / 1)$.

Note que ambos os modelos (i) e (ii) envolvem hipóteses que podem comprometer suas aplicações no sistema em estudo. Por exemplo, o modelo (i) admite que o sistema se comporte aproximadamente como um sistema de fila única, enquanto que, na prática, as filas se formam na frente de cada caixa. Por outro lado, o modelo (ii) admite que o sistema se comporte aproximadamente como $m$ sistemas de filas independentes, ou seja, o modelo supõe que, uma vez que um consumidor tenha entrado em uma das filas, ele não troque mais de fila. O que se observa na prática é que a troca de filas ocorre com freqüência. Note também que os modelos (i) e (ii) não consideram que os consumidores podem desistir de comprar, se as filas estiverem muito grandes.

A grande vantagem dos modelos (i) e (ii) é a simplicidade de análise, uma vez que os modelos $M / M / 1$ e $M / M / m$ são modelos Markovianos de fácil análise exata (veja, p.e., Kleinrock, 1975, Gross e Harris, 1974). Diversas medidas de desempenho podem ser facilmente computadas admitindo-se que o sistema esteja em equilíbrio, tais como a utilização média dos caixas, o número médio de consumidores no sistema, o tamanho médio da fila, o tempo médio de espera em fila, etc. Por exemplo, o tempo médio de espera em fila $W_{q}$ é determinado simplesmente por:

$$
\begin{aligned}
& W_{q}=\frac{\lambda / m}{\mu(\mu-\lambda / m)} \\
& W_{q}=\frac{(\lambda / \mu)^{m} p(0)}{\mu m(1-\rho)^{2} m !}
\end{aligned}
$$


onde: $\quad \rho=\frac{\lambda}{m \mu}$ e $p(0)=\left\{\sum_{i=0}^{m-1} \frac{(\lambda / \mu)^{i}}{i !}+\frac{(\lambda / \mu)^{m}}{(1-\rho) m !}\right\}^{-1}$

Um modelo Markoviano mais preciso para o presente problema do que os modelos (i) e (ii), aqui denominado modelo (iii), pode ser construído definindo-se o espaço de estados do problema como $\left(n_{1}, n_{2}, \ldots, n_{m}\right)$, onde $n_{i}$ denota o número de consumidores (em fila e serviço) no caixa $i, i=1, . ., m$. No caso dos supermercados, é razoável considerar que, a partir de um certo número $N$ de consumidores (em fila e serviço) em cada caixa, os novos consumidores, ao chegarem, desistem de entrar na fila e saem do supermercado sem comprar. Por exemplo, num sistema com $m=3$ e $N=3$, se um consumidor chega quando o sistema está no estado $(3,3,3)$, então este consumidor desiste de comprar e sai do supermercado. Assim, temos que $0 \leq n_{i} \leq N$ deve ser satisfeito para todo caixa $i$.

Em supermercados com um número de caixas não muito grande, digamos $m<10$, é razoável supor que os consumidores, ao chegarem, entram na fila de menor tamanho. Também é razoável supor que, após um certo tempo, se duas filas tiverem uma diferença de mais de $k$ consumidores, digamos $k=1,2$ ou 3, os consumidores da fila maior mudam para a fila menor (neste caso consideramos que a mudança ocorra instantaneamente). Desta forma, o espaço de estados do problema passa a ser definido por:

$\left(n_{1}, n_{2}, \ldots, n_{m}\right)$, com: $0 \leq n_{i} \leq N,\left|n_{i}-n_{j}\right| \leq k, i \neq j, i, j=1, . ., m$.

As possíveis transições entre estados ocorrem nos instantes em que: (i) um novo consumidor chega e entra no sistema, (ii) um consumidor termina de ser atendido por um caixa, o que pode envolver também alguma troca (instantânea) de filas. Sejam $\lambda$ a taxa média de chegada de consumidores no sistema, $\mu$ a taxa média de serviço (capacidade) de cada caixa, ambos definidos conforme anteriormente, e $\left(n_{1}, n_{2}, \ldots, n_{m}\right)$ o estado atual do sistema.

No caso (i), o sistema muda do estado atual:

$\left(n_{1}, n_{2}, \ldots, n_{i}, \ldots, n_{m}\right), n_{i}<N$

para o novo estado:

$\left(n_{1}, n_{2}, \ldots, n_{i}+1, \ldots, n_{m}\right)$

com taxa de transição $\lambda / m^{\prime}$, se $\left|n_{i}+1-n_{j}\right| \leq k$ para todo $j \neq i, j=1, . ., m$. O denominador $m$ ', $m$ ' $\leq m$, representa o número de possíveis transições a partir do estado atual $\left(n_{1}, n_{2}, \ldots, n_{m}\right)$, devido a chegada e entrada de um novo consumidor no sistema. Por exemplo, num sistema com $m=3, k=1$ e $N=3$, se o estado atual for $(1,0,0)$, o número de possíveis transições a partir de $(1,0,0)$ devido a uma chegada de um novo consumidor é $m^{\prime}=2$, uma vez que o sistema pode mudar apenas para os estados $(1,1,0)$ e $(1,0,1)$ (com taxa de transição $\lambda / 2$ para cada um). Note que, dado que $k=1$, o sistema não pode mudar para o estado $(2,0,0)$. Genericamente, $m$ ' pode ser definido por:

$m^{\prime}=\sum_{i=1, ., m} \delta\left(\left|n_{i}+1-n_{j}\right| \leq k\right)$

onde

$\delta\left(\left|n_{i}+1-n_{j}\right| \leq k\right)=\quad 1, \quad$ se $\left|n_{i}+1-n_{j}\right| \leq k$, para todo $j \neq i, j=1, . ., m$.

0 , caso contrário. 
No caso (ii), temos que considerar dois subcasos. No primeiro subcaso, temos que:

$\sum_{j=1, . ., m} \phi\left(n_{j}\right)<m$

onde

$\phi\left(n_{j}\right)=1, \quad$ se $n_{j}>0$

$0, \quad$ se $n_{j}=0$.

Note que, neste subcaso, há pelo menos um $n_{j}=0$. Desta forma, o sistema muda do estado atual:

$\left(n_{1}, n_{2}, \ldots, n_{i}, \ldots, n_{m}\right), n_{i}>0$

para o novo estado:

$\left(n_{1}, n_{2}, \ldots, n_{i}-1, \ldots, n_{m}\right)$

com taxa de transição $\mu$, se $\left|n_{i}-1-n_{j}\right| \leq k$, para todo $j \neq i, j=1, . ., m$. Por exemplo, num sistema com $m=3, k=1$ e $N=3$, se o estado atual for $(1,1,0)$, o sistema pode mudar apenas para os estados $(0,1,0)$ e $(1,0,0)$, com taxa de transição $\mu$ para cada um (obviamente não pode mudar para o estado $(1,1,-1))$.

O segundo subcaso é aquele em que:

$\sum_{j=1, \ldots, m} \phi\left(n_{j}\right)=m$

isto é, todos os $n_{j}$ são não nulos. Desta forma, o sistema muda do estado atual:

$\left(n_{1}, n_{2}, \ldots, n_{i}, \ldots, n_{m}\right)$

para o novo estado:

$\left(n_{1}, n_{2}, \ldots, n_{i}-1, \ldots, n_{m}\right)$

com taxa de transição $m \mu / m$ ”, se $\left|n_{i}-1-n_{j}\right| \leq k$, para todo $j \neq i, j=1, . ., m$. Similarmente ao caso (i), o denominador $m$ ", $m$ " $\leq m$, representa o número de possíveis transições a partir do estado atual $\left(n_{1}, n_{2}, \ldots, n_{m}\right)$, devido a uma saída de um consumidor do sistema. Por exemplo, num sistema com $m=3, k=1$ e $N=3$, se o estado atual for $(2,1,1)$, o número de possíveis transições a partir de $(2,1,1)$ devido a uma saída de um consumidor é $m$ "” $=1$, uma vez que o sistema pode mudar apenas para o estado $(1,1,1)$ (com taxa de transição $3 \mu$ ). Note que, dado que $k=1$, o sistema não pode mudar para os estados $(2,0,1)$ ou $(2,1,0)$, uma vez que admitimos que os consumidores mudam de fila instantaneamente. Genericamente, temos que:

$m ”=\sum_{i=1, ., m} \delta\left(\left|n_{i}-1-n_{j}\right| \leq k\right)$

onde

$\delta\left(\left|n_{i}-1-n_{j}\right| \leq k\right)=\quad 1, \quad$ se $\left|n_{i}-1-n_{j}\right| \leq k$, para todo $j \neq i, j=1, . ., m$.

0, caso contrário. 
Escrevendo-se as equações de equilíbrio para cada possível estado do sistema $\left(n_{1}, n_{2}, \ldots, n_{m}\right)$, com: $n_{i} \leq N,\left|n_{i}-n_{j}\right| \leq k, i \neq j, i, j=1, . ., m$, e substituindo-se uma destas equações pela equação representando a soma das probabilidades de todos os estados igual a 1 (para evitar um sistema indeterminado), obtemos um sistema linear com, no máximo, $(N+1)^{m}$ equações e incógnitas (lembre-se que temos a condição adicional $\left|n_{i}-n_{j}\right| \leq k$ que, dependendo do valor de $k$, reduz o tamanho do sistema). Note que, dados $N$ e $k$, este sistema cresce exponencialmente com o número de caixas $m$.

Por exemplo, para um problema com $m=3, k=1$ e $N=3$, o sistema resulta em 22 equações, bem menor do que o limitante acima $(N+1)^{3}=64$. Aumentando-se $m$ para 4 e 5 , obtemos sistemas com 46 e 94 equações, respectivamente, também bem menores que $(N+1)^{4}=256$ e $(N+1)^{5}=1024$, mas ainda com crescimento exponencial. A figura 2 ilustra parte do diagrama de transição de estados para o problema com $m=3, k=1$ e $N=3$.

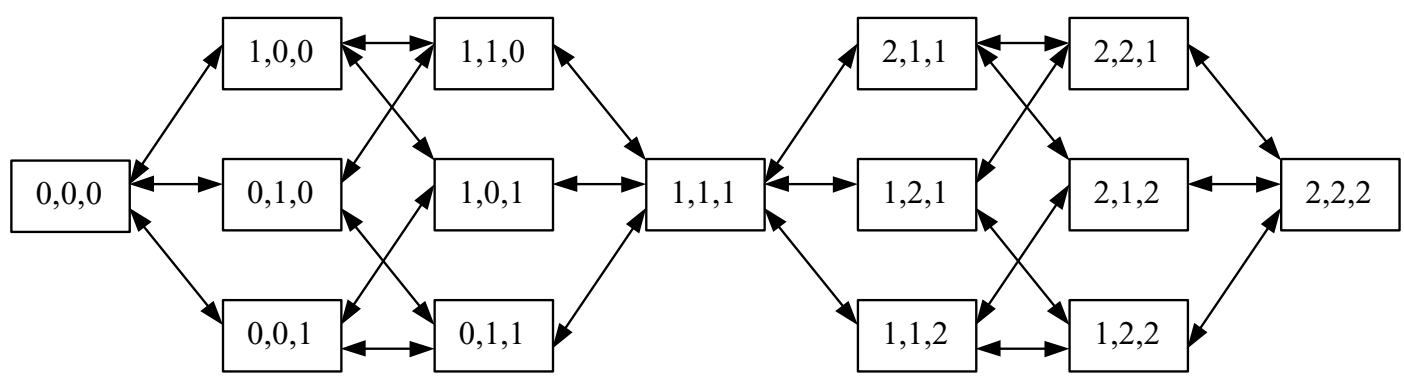

Figura 2 - Parte do diagrama de transição de estados para o problema com $m=3, k=1$ e $N=3$.

Resolvendo-se o sistema linear, obtemos $p\left(n_{1}, n_{2}, \ldots, n_{m}\right)$, a distribuição de equilíbrio dos estados do sistema. Tomemos, por exemplo, os estados $(0,0,1)$ e $(2,2,1)$ da figura 2 . Note que, seguindo as definições dos casos (i) e (ii) discutidos acima, as equações de equilíbrio para estes estados resultam em:

Estado $(0,0,1):(\lambda / 2+\lambda / 2+\mu) p(001)=\lambda / 3 p(000)+\mu p(101)+\mu p(011)$

Estado $(2,2,1):(\lambda+3 \mu / 2+3 \mu / 2 p(221)=\lambda / 2 p(121)+\lambda / 2 p(211)+3 \mu / 3 p(222)$

(o sistema linear completo para o caso em que $\lambda=\mu=1$ encontra-se no anexo). Com a distribuição $p\left(n_{1}, n_{2}, \ldots, n_{m}\right)$, podemos facilmente computar medidas de desempenho do sistema, por exemplo, o workload do caixa $i$ é calculado simplesmente por:

$\rho_{i}=\sum_{\left(n_{1}, n_{2}, \ldots, n_{m}\right) \mid n_{i}>0} p\left(n_{1}, n_{2}, \ldots, n_{m}\right)$

e o tempo médio de espera na fila do caixa $i$ é facilmente obtido por:

$W q_{i}=\sum_{\left(n_{1}, n_{2}, \ldots, n_{m}\right) \mid n_{i}>0} n_{i} p\left(n_{1}, n_{2}, \ldots, n_{m}\right) /(\lambda / m)-\frac{1}{\mu}$

Convém observar que os modelos (i) e (ii) podem ser vistos como casos particulares do modelo (iii). Pode ser mostrado que o modelo (i), com um sistema $M / M / m$ com fila única, é o caso particular do modelo (iii) quando $k=1$ e $N=$ infinito. Por outro lado, o modelo (ii), com $m$ sistemas de fila $M / M / 1$, é o caso particular do modelo (iii) quando $k=$ infinito e $N=$ infinito. Neste 
trabalho não são explorados modelos baseados em um sistema $M / M / m / N$ com fila única, ou $m$ sistemas $M / M / 1 / N$ paralelos e independentes, uma vez que estes também podem ser vistos como casos particulares do modelo (iii) quando $k=1$ e $k=$ infinito, respectivamente. Os resultados obtidos com a aplicação dos três modelos para os dados do estudo de caso da próxima seção são comparados e analisados na seção 5.

\section{Estudo de caso}

Um estudo de caso foi realizado num supermercado da rede Jaú-Serve, localizado em São Carlos, SP. Esta rede vem crescendo nos últimos anos no interior de São Paulo, com consumidores principalmente das classes média e baixa. As lojas vem sendo modernizadas, com check-outs com leitura ótica, consultores de preço eletrônicos, etc., e procuram avaliar a credibilidade e fidelidade dos seus consumidores por meio de pesquisas de opinião.

Para absorver o acréscimo de demanda nos dias/horários mais congestionados, o gerente do supermercado conta com funcionários que normalmente não trabalham nos caixas durante os outros dias/horários, mas que são capazes de desempenhar essa função quando necessário. Nos demais dias/horários, o supermercado opera com um número menor de caixas, e costuma abrir um novo caixa assim que o número de clientes nas filas começa a ficar maior do que três.

Coletamos uma amostra num sábado, das 14:00 às 18:00 hs. Durante este período, o sistema operou com 5 caixas normais (caixas $1,2, \ldots, 5$ ) e 2 caixas rápidos (caixas 6 e 7). Para cada caixa, foram coletados os instantes de chegadas de consumidores na fila, de eventuais desistências durante a espera, e de início e término de serviços (ordem de centenas de observações). A tabela 1 apresenta os intervalos médios entre chegadas e os tempos médios de serviço dos consumidores para cada caixa. A média geral dos intervalos médios entre chegadas nos caixas normais é 7,0 minutos (i.e., 0,14 chegadas por minuto em cada caixa, ou $\lambda=0,71$ ), e nos caixas rápidos é 3,5 minutos $(0,29$ chegadas por minuto em cada caixa, ou $\lambda=0,57)$. $\mathrm{A}$ média geral dos tempos médios de serviço nos caixas normais é 4,6 minutos (i.e., $\mu=0,22$ atendimentos por minuto em cada caixa), e nos caixas rápidos é 1,55 minutos $(\mu=0,64$ atendimentos por minuto em cada caixa). A última coluna da tabela apresenta os tempos médios de espera em fila obtidos pela amostra (e não pelos modelos)

Tabela 1 - Intervalos médios entre chegadas e tempos médios de serviço dos consumidores nos caixas

\begin{tabular}{cccc}
\hline Caixa & $\begin{array}{c}\text { Intervalo médio } \\
\text { Entre chegadas (min) }\end{array}$ & $\begin{array}{c}\text { Tempo médio de } \\
\text { serviço (min) }\end{array}$ & $\begin{array}{c}\text { Tempo médio de } \\
\text { espera em fila (min) }\end{array}$ \\
\hline 1 & 8,6 & 5,6 & 3,2 \\
2 & 6,5 & 4,3 & 1,9 \\
3 & 5,8 & 4,5 & 1,6 \\
4 & 7,1 & 4,8 & 2,5 \\
5 & 6,6 & 4,2 & 1,6 \\
Média & 7,0 & 4,6 & 2,16 \\
Desvio Padrão & 1,06 & 0,56 & \\
& & & 0,9 \\
6 & 3,3 & 1,6 & 1 \\
7 & 3,7 & 1,5 & 0,95 \\
Média & 3,5 & 1,55 & \\
Desvio Padrão & 0,29 & 0,07 & \\
\hline
\end{tabular}


Para cada tipo de caixa testamos, por meio de análise de variância, as hipóteses de que as médias dos intervalos de tempo entre chegadas eram iguais. Em ambos os casos, não foi possível rejeitar estas hipóteses com 5\% de significância. Também testamos as hipóteses de que as médias dos tempos de serviço eram iguais para os caixas normais e rápidos, e, em ambos os casos, também não foi possível rejeitar tais hipóteses com 5\% significância. Observe que, para cada tipo de caixa, os desvios padrões dos intervalos de tempo entre chegadas e dos tempos de serviço são bem pequenos, comparativamente às suas respectivas médias (tabela 1). Assim, é razoável admitir que todos os caixas normais e todos os caixas rápidos têm intervalos médios entre chegadas e tempos médios de serviço iguais. A tabela 2 apresenta as taxas e as utilizações médias correspondentes.

Tabela 2 - Taxas e utilizações médias dos caixas normais e rápidos

\begin{tabular}{cccc}
\hline Tipo de caixa & $\begin{array}{c}\lambda \\
\text { (clientes/min) }\end{array}$ & $\begin{array}{c}\mu \\
\text { (clientes/min) }\end{array}$ & $\rho=\lambda / m \mu$ \\
\hline Normal & 0,71 & 0,22 & 0,65 \\
Rápido & 0,57 & 0,64 & 0,45 \\
\hline
\end{tabular}

Para verificar as hipóteses da seção 3 de que os intervalos de tempo entre chegadas e os tempos de serviço são exponencialmente distribuídos, aplicamos os testes de Kolmogorov-Smirnov e Anderson-Darling (utilizamos o software Best-Fit 2.0d da Palisade Tools). Os testes não rejeitaram (5\% de significância) a hipótese de intervalos entre chegadas exponenciais em todos os caixas, exceto no caixa normal 4. No caso dos tempos de serviço, os testes não rejeitaram a hipótese de distribuição exponencial em todos os caixas, exceto nos caixas rápidos 6 e 7 . Entretanto, por simplicidade, admitimos que os intervalos entre chegadas e os tempos de serviço sejam exponencialmente distribuídos em todos os caixas, com taxas médias iguais às da tabela 2. Desta maneira, os três modelos discutidos na seção anterior podem ser utilizados como aproximações.

\section{Medidas de desempenho}

Nesta seção analisamos os resultados obtidos com a aplicação do modelo (i) (um sistema $M / M / m$ de fila única), modelo (ii) ( $m$ sistemas $M / M / 1$ paralelos e independentes), e modelo (iii) da seção 3. Neste último, admitimos que $N=5$ para os caixas normais, e $N=10$ para os caixas rápidos. A escolha destes valores levou em conta o espaço disponível para formação de filas na frente dos caixas, e o fato dos consumidores dos caixas normais em geral utilizarem carrinhos grandes, ao contrário dos consumidores dos caixas rápidos. Conforme o gerente do supermercado, filas com mais de três consumidores em todos os caixas normais começam a gerar reclamações por parte dos consumidores.

Os modelos (i)-(iii) foram implementados em linguagem Pascal (compilador Delphi 4) num microcomputador Pentium $100 \mathrm{Mhz}$. Os sistemas lineares do modelo (iii) foram gerados automaticamente, conforme as idéias de Larson (1974) para o modelo hipercubo, e resolvidos por meio do método de Gauss-Jordan. Os tempos computacionais para resolvê-los foram da ordem de poucos segundos.

As tabelas 3 e 4 apresentam o número médio de consumidores no sistema e o tempo médio de espera em fila obtidos com cada um dos três modelos para os caixas normais $(m=5)$ e rápidos $(m=2)$, respectivamente. Por conveniência, a última coluna das tabelas apresenta novamente o tempo médio de espera em fila observado na amostra coletada, conforme tabela 1. No caso do 
modelo (iii), as tabelas 3 e 4 apresentam os valores obtidos variando-se $k$ a partir de 1, até que os tempos médios de espera em fila $W_{q}$ fossem maiores do que os observados na amostra (última coluna da tabela 1). Lembre-se da seção 3 que os tempos médios de espera em fila para os modelos (i) e (ii) são casos especiais do modelo (iii) com $k=1$ e $N=$ infinito, e $k=$ infinito e $N=$ infinito, respectivamente.

Tabela 3 - Número médio de consumidores no sistema e tempo médio de espera em fila para caixas normais

\begin{tabular}{|c|c|c|c|c|}
\hline & $\begin{array}{l}\text { Modelo (i): } \\
\text { M/M/5 }\end{array}$ & $\begin{array}{l}\text { Modelo (ii): } \\
{[\mathrm{M} / \mathrm{M} / 1]^{5}}\end{array}$ & Modelo (iii) & $\begin{array}{c}\text { Valor observado } \\
\text { na amostra }\end{array}$ \\
\hline Número médio de & 3,77 & 9,10 & $3,77(\mathrm{k}=1)$ & \\
\hline Consumidores no sistema & & & $4,98(\mathrm{k}=2)$ & \\
\hline Número médio de & 0,75 & 1,82 & $0,75(\mathrm{k}=1)$ & \\
\hline Consumidores por caixa & & & $1,00(\mathrm{k}=2)$ & \\
\hline Tempo médio de & 0,76 & 8,28 & $0,76(\mathrm{k}=1)$ & 2,16 \\
\hline Espera em fila (min) & & & $2,47(\mathrm{k}=2)$ & \\
\hline $\begin{array}{l}\text { Desvio em relação } \\
\text { ao observado }(\%)\end{array}$ & $-65 \%$ & $283 \%$ & $\begin{array}{c}-65 \%(\mathrm{k}=1) \\
14 \%(\mathrm{k}=2)\end{array}$ & $0 \%$ \\
\hline
\end{tabular}

Tabela 4 - Número médio de consumidores no sistema e tempo médio de espera em fila para caixas rápidos

\begin{tabular}{lcccc}
\hline & $\begin{array}{c}\text { Modelo (i): } \\
\mathrm{M} / \mathrm{M} / 2\end{array}$ & $\begin{array}{c}\text { Modelo (ii): } \\
{[\mathrm{M} / \mathrm{M} / 1]^{2}}\end{array}$ & Modelo (iii) & $\begin{array}{c}\text { Valor observado } \\
\text { na amostra }\end{array}$ \\
\hline Número médio de & 1,11 & 1,61 & $1,11(\mathrm{k}=1)$ & \\
Consumidores no sistema & & & $1,29(\mathrm{k}=2)$ & \\
& & & $1,42(\mathrm{k}=3)$ & \\
& & & $1,50(\mathrm{k}=4)$ & \\
Número médio de & 0,56 & 0,80 & $0,56(\mathrm{k}=1)$ & \\
Consumidores por caixa & & & $0,65(\mathrm{k}=2)$ & \\
& & & $0,71(\mathrm{k}=3)$ & \\
Tempo médio de & & $0,75(\mathrm{k}=4)$ & 0,95 \\
espera em fila (min) & 0,39 & 1,25 & $0,39(\mathrm{k}=1)$ & \\
& & & $0,70(\mathrm{k}=2)$ & \\
& & & $0,93(\mathrm{k}=3)$ & \\
Desvio em relação & & $1,07(\mathrm{k}=4)$ & \\
ao observado (\%) & & & $-59 \%(\mathrm{k}=1)$ & \\
& $-59 \%$ & & $-26 \%(\mathrm{k}=2)$ & \\
& & & $-2 \%(\mathrm{k}=3)$ & \\
& & & $13 \%(\mathrm{k}=4)$ & \\
\hline
\end{tabular}

Como era esperado (conforme discussão na seção 3), tanto para os caixas normais quanto para os rápidos, os tempos médios de espera mais próximos dos observados na amostra foram obtidos com o modelo (iii). Nos caixas normais (tabela 3), o valor 2,47 minutos foi obtido com $k=2$ (com um desvio de $14 \%$ do valor observado 2,16 minutos), enquanto que nos caixas rápidos (tabela 4), o valor 0,93 minutos foi obtido com $k=3$ (desvio de apenas $-2 \%$ em relação ao observado 0,95 minutos). Os sistemas lineares correspondentes resultaram em 876 e 65 equações, respectivamente. A precisão da aproximação fornecida pelo modelo (iii) é aceitável para tomar decisões de alocação de capacidade em função do nível de serviço desejado para os consumidores (no caso, o tempo médio de espera em fila). Isto sugere que o modelo (iii), 
diferente dos modelos (i) e (ii), pode ser uma boa aproximação para o sistema de filas do supermercado em consideração.

Convém observar que, na amostra em consideração, os consumidores dos caixas rápidos parecem ser um pouco mais tolerantes a variações nos tamanhos das filas dos caixas adjacentes do que os consumidores dos caixas normais $(k=2$, no caso dos caixas normais, contra $k=3$, no caso dos caixas rápidos). Isto talvez seja devido ao fato de que os tempos médios de espera em fila e os tempos médios de serviço nos caixas rápidos sejam bem menores do que nos caixas normais. Acreditamos que isso nem sempre ocorre, mesmo porque esperávamos o efeito oposto, devido ao fato dos caixas normais serem mais numerosos ( $m=5$ caixas normais, contra $m=2$ caixas rápidos) e seus consumidores carregarem bem mais itens de compra, o que poderia desestimular os consumidores em fila a mudarem para filas mais distantes. Experimentos adicionais precisariam ser realizados para verificar melhor estas observações; note que, no presente experimento, a hipótese de distribuição exponencial para os tempos de serviço dos caixas rápidos foi rejeitada (seção 4), o que pode comprometer parte das observações acima, baseadas nos resultados de um modelo Markoviano.

Uma última observação é que os resultados do modelo (iii) são robustos em relação à pequenas variações no parâmetro $N$. Por exemplo, variando-se $N$ dos caixas normais em mais ou menos $20 \%$ (i.e., de $N=5$ para $N=6$, ou de $N=5$ para $N=4$ ), o tempo médio de espera em fila varia menos de $0,5 \%$ (i.e., de 2,47 para 2,48 minutos, ou de 2,47 para 2,46 minutos, respectivamente).

\section{Conclusões e perspectivas}

Neste estudo aplicamos teoria de filas para modelar o tempo médio de espera dos consumidores nos caixas de supermercados. Três modelos foram explorados: (i) representar o sistema por meio de um modelo $M / M / m$ de fila única, onde $m$ é o número de caixas em operação, (ii) representar o sistema por meio de $m$ modelos $M / M / 1$ paralelos e independentes, e (iii) representar o sistema por meio de um modelo Markoviano mais geral, onde os dois modelos anteriores podem ser vistos como casos particulares. Para avaliar o desempenho destes modelos, realizamos um estudo de caso num dos supermercados da rede Jaú-Serve, que atua no interior de São Paulo. Os resultados mostraram que o modelo (iii) pode ser uma boa aproximação para o sistema, ao contrário dos modelos (i) e (ii).

Acreditamos que outros experimentos ainda são necessários para avaliar a precisão do modelo (iii) e sua validação nos casos práticos. Uma perspectiva interessante para pesquisa futura é investigar a sensibilidade do modelo com respeito a variações no valor dos parâmetros, e explorar o projeto ótimo e a análise de curvas de tradeoff entre medidas de desempenho do sistema, a exemplo do que foi feito em Tang e Yoo (1991), Fontanella e Morabito (1997) e Bitran e Morabito (1999). Em particular, poderia-se estudar melhor o tradeoff entre o nível de serviço (p.e., tempo médio de espera em fila) e a capacidade do sistema (número de caixas normais e rápidos num dado período).

Outra perspectiva interessante para pesquisa futura é desenvolver aproximações baseadas no modelo (iii) para os casos em que o número de caixas $m$ é grande (lembre-se da seção 3 que o número de equações de equilíbrio do modelo cresce exponencialmente com o aumento de $m$ ). Nestes casos, o modelo (iii) deve ser adaptado para tratar situações em que os consumidores, ao chegarem, escolhem o caixa com menor fila apenas dentre os caixas mais próximos ou dentro do seus campos de visão, ao invés de todos os caixas. Similarmente, as situações em que os consumidores mudam de fila dependendo do tamanho das filas apenas dos caixas adjacentes, ao invés de todos os caixas. 


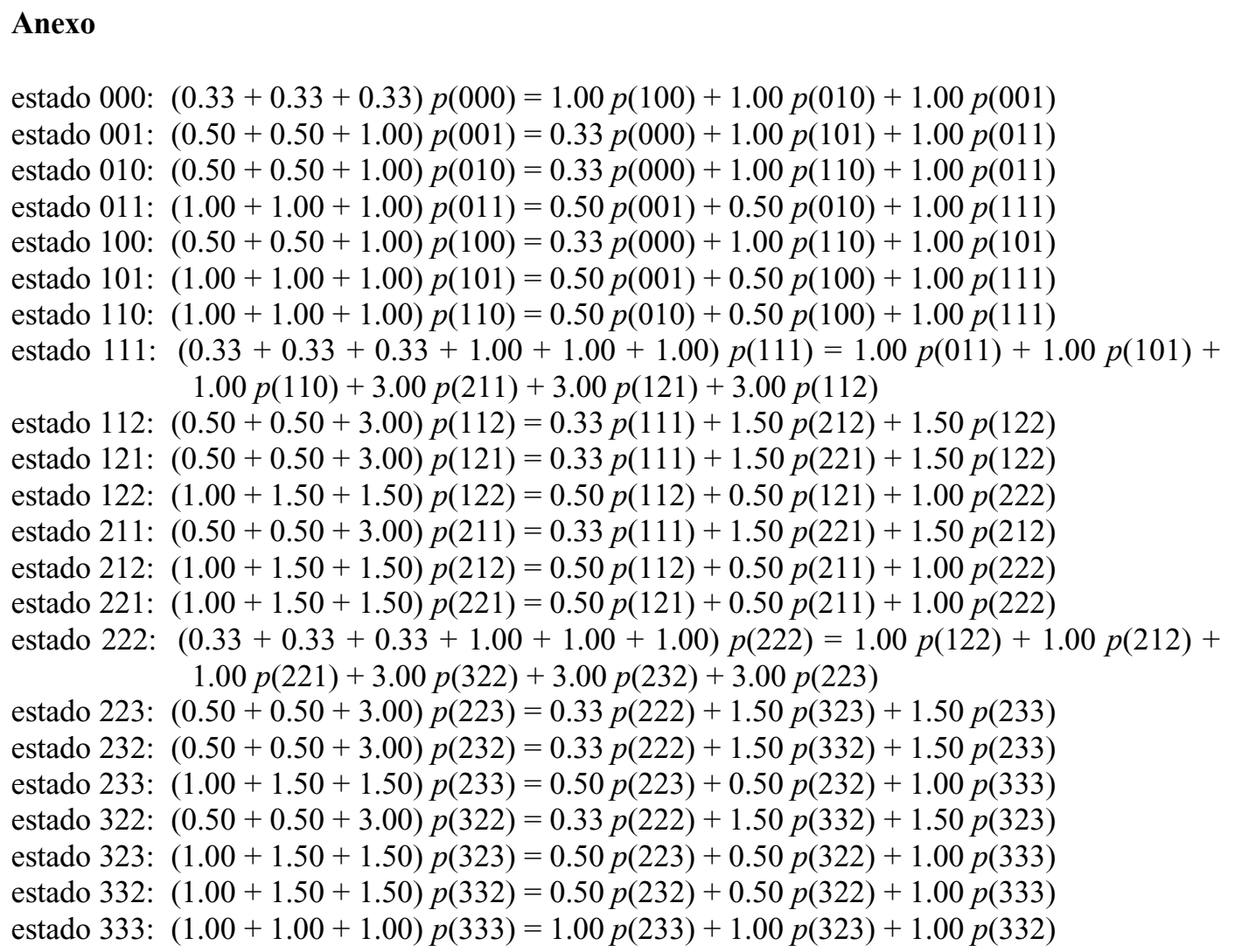

\section{Agradecimentos}

Agradecemos à Maria Creusa B. Salles do ICMC-USP e aos revisores anônimos pelos úteis comentários e sugestões. Também agradecemos ao Rodolfo Donizetti Goffredo do Hipermercado Jaú-Serve de São Carlos, por sua colaboração para que a coleta de dados pudesse ter sido realizada.

\section{Referências}

Andersen (1997). “Andersen Consulting's marketing retail in Latin American". Technical Report, Andersen Consulting, São Paulo, SP, abril, 86-93.

Bitran, G. R. e Morabito, R. (1999). "An overview of tradeoff curves in manufacturing system design". Production and Operations Management v.8, n.1, 56-75.

Dijk, N. M. (1997). "Why queuing never vanishes". European Journal of Operational Research, v.99, n.2

Fontanella, G. e Morabito, R. (1997). "Modelagem por meio de teoria de filas do tradeoff entre investir em canais de atendimento e satisfazer o nível de serviço em provedores internet", Gestão \& Produção, 4(3), 278-295. 
Gross, D. e Harris, C. M. (1974). Fundamentals of queueing theory. John Wiley \& Sons, New York.

Kleinrock, L. (1975). Queueing systems, vol.1: computer applications. John Wiley \& Sons, New York.

Larson, R. C. (1974). "A hypercube queuing model for facility location and redistricting in urban emergency services". Computers \& Operations Research, vol.1, 67-95.

OMC/EAN (1996). "A percepção do consumidor para com os sistemas de automação em supermercados". Relatório Técnico, Organon Marketing Consulting / EAN Sistemas do Brasil, São Paulo, SP, agosto.

Superhiper (1998). Revista SuperHiper, Associação Brasileira de Supermercados (ABRAS), ano 24, n.277, São Paulo, SP, setembro.

Tang, C. S. e Yoo, S. (1991). "System planning and configuration problems for optimal system design". European Journal of Operational Research 54, 163-175.

Umesh, U., Pettit, K. e Bozman, C. (1989). "Shopping model of the time-sensitive consumer". The Journal of Decision Sciences Institute, v.20, n.4, 715-789. 